\title{
An lloT-based architecture for decision support in the aeronautic industry
}

\author{
Roberto Vita ${ }^{1}$, Narciso Caldas ${ }^{1}$, João Basto ${ }^{1}$, Symone Alcalá ${ }^{2}$, and Flavio Diniz ${ }^{3}$ \\ ${ }^{1}$ Center for Enterprise Systems Engineering, INESC TEC, 4200-425 Porto, Portugal \\ ${ }^{2}$ Faculty of Science and Technology, Federal University of Goiás, 74968-755, Goiás, Brazil \\ ${ }^{3}$ Embraer SA, Avenida Brigadeiro Faria Lima, 2170, CEP 12227-901, São José dos Campos, Brazil
}

\begin{abstract}
The Industry 4.0 movement is driving innovation in manufacturing through the application of digital technologies, leading to solid performance improvements. In this context, this paper introduces a real-time analytical framework based on predictive, simulation and optimization technologies applied to decision support in manufacturing systems, enabled by an underlying reference implementation of an open Industrial Internet of Things (IIOT) platform. This architecture integrates critical equipment, manufacturing and corporate systems through a Unified IIOT Cloud Platform. A real case study on the aeronautic industry demonstrates the proposal feasibility of this architecture to enhance productivity, predict equipment failures and bring agility to react to unexpected events. In this case study, the monitoring tool displays the current status of the critical resources and the predictive tool calculates a probability of failure. When this probability reaches a certain threshold, the simulation tool is triggered to evaluate the impact of the disruption in the system's productivity. Results from the tools are displayed online through an alert system so that each stakeholder is informed timely and in a contextualized way.
\end{abstract}

\section{Introduction}

Nowadays, industries need to improve performance, either due to the market demand or to the technological forces driven by the Industry 4.0 growth. In Industry 4.0, smart manufacturing is the key element. It has the power to increase flexibility, productivity and quality, and helps to create customized products at large scale. Moreover, Industry 4.0 relies on the use of digital technologies, which produce and process data in real time, providing useful information to the manufacturing system for decision-making support [1]. Technologies, such as Internet of Things (IoT), big data, simulation, optimization and artificial intelligence are part of the development of smart manufacturing systems.

In particular, an IoT ecosystem consists of a network of objects (such as sensors, tablets, machines, among others) that allows interaction and communication with each other. Modern factories, by capturing and combining data from many IoT objects, potentiate the development and use of advanced tools, to support the decision-making process and improve performance by monitoring processes in real-time, predicting equipment faults, optimizing flexible lines and implementing autonomous processes [2]. 
Different decision support systems (DSS) have been proposed in literature, however few combine Data Science, Simulation and Optimization tools for online diagnostic and planning. Additionally, having a system with the ability to monitor the shop floor in realtime enables companies to behave fast and react timely to constantly changing demands.

This paper addresses one of the manufacturing digital transformation trends by presenting a DSS framework with real-time monitoring capabilities that considers a hybrid approach of predictive, simulation and optimization technologies connected through an open industrial IoT (IIoT) platform. A real case study on the aeronautic industry demonstrates the proposal feasibility of this architecture to enhance productivity, predict equipment failures and bring agility to react to unexpected events.

Section 2 introduces proper concepts of IoT technologies and DSS. Section 3 presents the decision support framework and the IIoT architecture. Section 4 describes the application case of the DSS framework on the aeronautic industry. The last section indicates some conclusions and remarks.

\section{Background}

The industry 4.0 technologies have enabled manufacturing companies to create intelligent systems that improve the decision-making process. In this context, the information systems that support business decision-making activities have received several advances [3].

DSS vary according to their objective, frequency of decision-making, degree of guidance, among other criteria [4]. [5] points out the importance of DSS on the design, scheduling and control of digital manufacturing networks. Additionally, according to [6], the different functions of DSS includes enabling access, visualization and manipulation of data; work cooperation; problem-solving through rules and algorithms; and the use of models for statistical, financial, optimization or simulation purposes.

The Simulation technology has been incorporated into DSS mainly to recreate a physical system in a digital format, in order to evaluate and analyse its stochastic behaviour [7]. Also, it allows evaluating different scenarios without compromising the real system, such as changing the production mix or adding new machines [8]. The Optimization technology is incorporated into DSS to help companies defining plans or strategies which can maximize or minimize a given objective function, while respecting a series of constraints [9]. In this sense, optimization can be used in a number of industry related decision-making problems, such as production scheduling [10] or supply chain design [11].

The use of machine learning in the manufacturing environment is not new [12]. However, with the technology advancement, it figures as a promising technology to generate value [13]. Data scientists are using data in order to obtain useful insights, to figure out root causes of problems, to make predictions, and then support decisions and improve productivity [14]. One application of this data-driven approach relies on asset management, which helps companies to achieve higher reliability and availability of equipment [15].

DSS also takes into account how users interact with the available data. In this case, dashboards and reports are the most used interfaces in the industry, which enable decision makers to monitor critical processes, explore data from multiple perspectives, and manage processes in order to improve decisions and system performance [16].

The integration of analytical and simulation models has the potential to create enhanced DSS. These hybrid models impel the use of commissioning-simulation methods not only to support periodic planning at the strategic and tactical level, but also to allow for data-driven execution decision-making in real time [17]. These new approaches will solve industrial problems with gains in flexibility, scalability and efficiency [18]. 
DSS are using Cyber Physical Systems to improve decision-making in a manufacturing facility and support automatic response with minimum human interaction [17].

The use of data-driven models raises the discussion about data acquisition, data processing, interoperability and the human-machine interaction [19]. In this environment, the manufacturing system is supported by an innovative platform, such as the IIoT Platform, that assists data management and services [20], and will generate an integrated and intelligent system [21].

These technological advancements have been revolutionizing the industrial environment. However, the success of companies depends on the capacity to identify, collect and analyse the right data in order to create value [22].

The Aeronautic industry has many variables, such as complex products, different fabrication processes, long development cycles, and long product lifecycles, which imposes challenges to ensure productivity, efficiency, quality, and production costs. An important factor to remain competitive is the ability to keep the operations running smoothly. Specifically, decisions regarding facility layout, production planning and scheduling, considering the necessity to react to minor daily problems, such as resources unavailability. This requires being able to simultaneously assess and analyse a huge amount of data and quickly act upon it, which is only feasible with the aid of modern DSS.

\section{IloT based architecture for decision support in manufacturing system}

The proposed IIoT architecture for decision support in manufacturing system, illustrated in Figure 1, is composed by a Decision-Support Framework called FASTEN Suite Tool, which is backed by an Open IIoT platform that ensures a clean bi-directional integration among the system components.

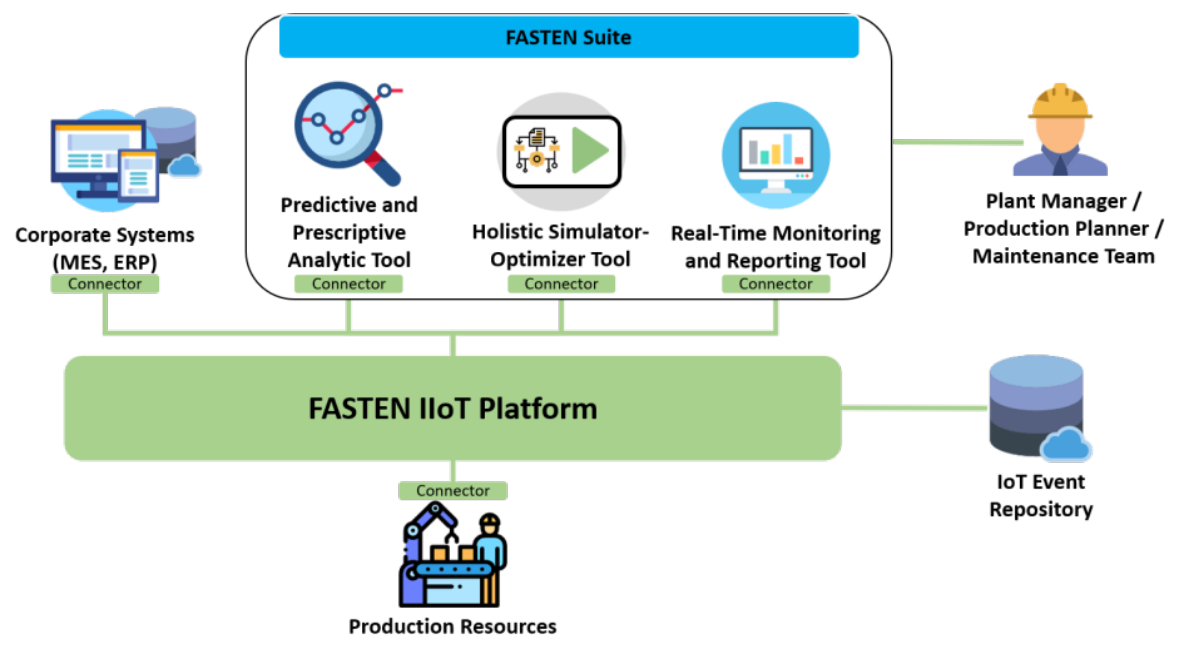

Fig. 1. IloT based architecture for decision support system.

This framework forms an infrastructure which is the pillar of the shop-floor digital transformation both in the technical and management aspects. It considers the integration of the FASTEN Suite Tool, corporate systems, and the production resources.

The communication between different systems and software modules is achieved by means of the IIoT Platform, which provides a broker subscription API (Application Programming Interface). 


\subsection{Decision support framework: Real-Time Monitoring Tool with predictive, optimization, simulation and visualization tools.}

The FASTEN Suite Tool is composed by a Simulator-Optimizer module; a Predictive and Prescriptive Analytics module; and a Real-Time Monitoring module. Depending on the use case, the tools can be used standalone or integrated.

The Simulator-Optimizer Tool combines discrete event simulation with linear programming and heuristics to create optimized solutions and test their behaviour in a virtual representation of the system. The Predictive and Prescriptive Analytics Tool encompasses an open source machine-learning library that allows deploying and managing predictive models. The Real-Time Monitoring Tool uses a set of data visualization tools to display data from the different components as dashboards and reports. In addition, it provides an interface where the user can interact with the tools.

The FASTEN Suite Tool data input comes from sensors (e.g. vibration, temperature, acceleration, among others) and from corporate systems (e.g. Manufacturing Execution System (MES), Maintenance Management System (MMS), among others). The sensory data acquired from the production processes are conditioned and pre-processed locally (e.g. sampling, filtering, compressing, etc.) before being sent to the IIoT platform. The different tools can publish and/or subscribe to message topics, in order to request or provide data. The IIoT Event Repository stores all the exchanged data in specific datasets.

This architecture provides relevant tools for the digital manufacturing transformation journey, including visibility, transparency and predictability capabilities, and even means to reach the highest maturity level of Industry 4.0, namely the autonomous adaptability.

\subsection{FASTEN Open IloT Architecture}

The IIoT platform reference architecture, in figure 2, is based on the RAMI 4.0 (Reference Architectural Mode for Industrie 4.0) [23]. This architecture supports collaboration and integration with other relevant initiatives by framing the developed concepts and technologies in a common model and is part of the FASTEN project development [24].

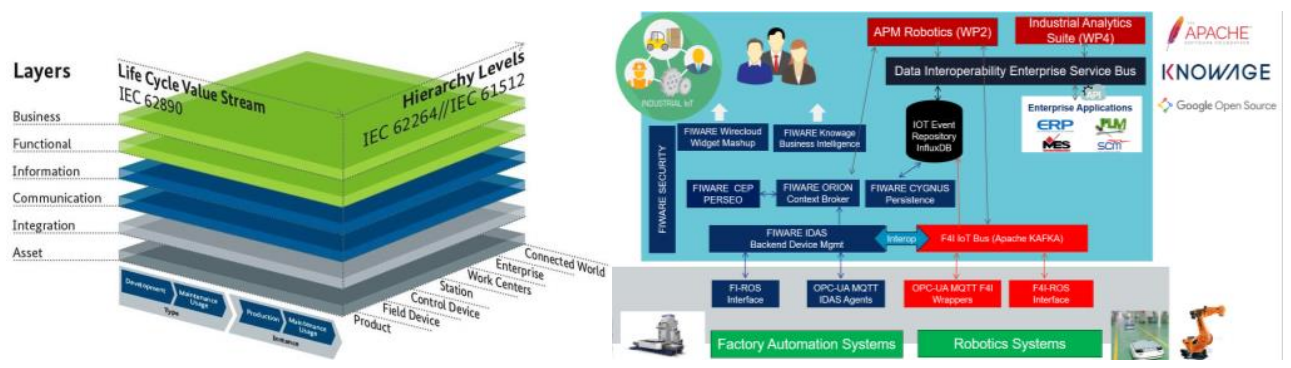

Fig. 2. RAM I 4.0 reference architecture [23] and the FASTEN IIOT Platform [24]

The FASTEN open reference architecture specifies different functional components and their interfaces. It has two alternative brokers to connect the components: a Kafka broker, on the Apache lane, and an Orion Context broker, on the FIWARE lane.

Both Apache and FIWARE lane are able to provide a message exchange mechanism between OPC UA sources (i.e. equipment) and the IIoT platform. The Apache lane uses a bidirectional MQTT to OPC UA Bridge. The FIWARE lane uses the OPC UA IoT Agent.

The IIoT database used to store historical data generated from the different FASTEN data sources in FIWARE lane is CrateDB. In Apache lane, the raw IIoT data is stored in Apache Kafka automatically and after processing the data, it is transferred to InfluxDB. 


\section{Application Case}

The FASTEN Suite was developed and applied on a use case of the Metallic Centre Wing Assembly Line (WAL) at the Embraer Aerospace facility in Évora, Portugal, where parts from internal and external production streams are combined to form complex structures.

The manufacturing process is partially automated, using both robotic resources and human operators. It produces different products that present specific tasks that can follow different sequences with different processing times. The line is susceptible to disturbances that affect the company's overall performance, such as unbalanced scheduling, product or process changes, equipment breakdown, and parts unavailability. The WAL operations involve high complexity decision-making and require high flexibility.

Thus, the FASTEN Suite is presented as a solution to enhance real-time support material for decision-making on shop floor and help managers to deal with unexpected events and evaluate production scenarios. It focuses on the improvement of the Overall Line Efficiency (OLE), Resource utilization, and Maintenance Costs by using the ability of line balancing, scheduling optimization, failure prediction, and performance simulation.

\subsection{Use Case Description}

In the considered use case, the production resources of the line continually send data to the IIoT Platform. The Predictive Tool gets and analyses equipment data to predict the probability of the resources failing, while the Real-Time Tool displays that probability and the most relevant information from the system. When such probability exceeds a threshold, an alarm is triggered and the Simulation and Optimization Tool is actioned in order to estimate the best time to execute the maintenance operations, based on the production and the maintenance system information. In addition, the simulation evaluates the impact on the system of making such a decision. Different scenarios can be generated in loop. The results are displayed on dashboards in the Real-Time Tool, and an e-mail is sent to the users notifying the availability of the results. The sequence diagram in Figure 3 presents the flow of events and communications between the different components in the use case.

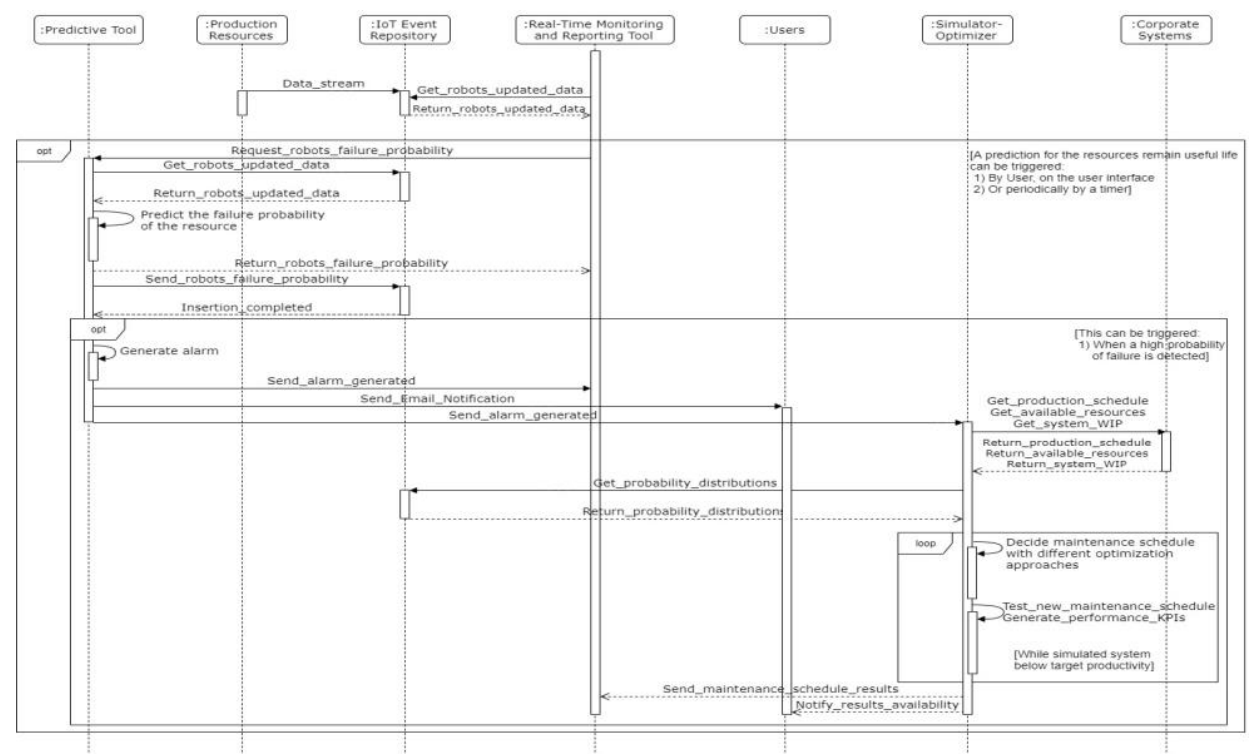

Fig. 3. U se case - Sequence diagram. 


\subsection{Data Model}

The data model of this case has manufacturing systems represented by a set of production lines. Each line contains workstations, buffers, and transport paths. Buffers and transport paths could be associated with workstations. The workstation has associated operations, which tasks depend on each product. The tasks can be performed by fixed or mobile resources and their historic times are registered. Both resources can be robotic or human.

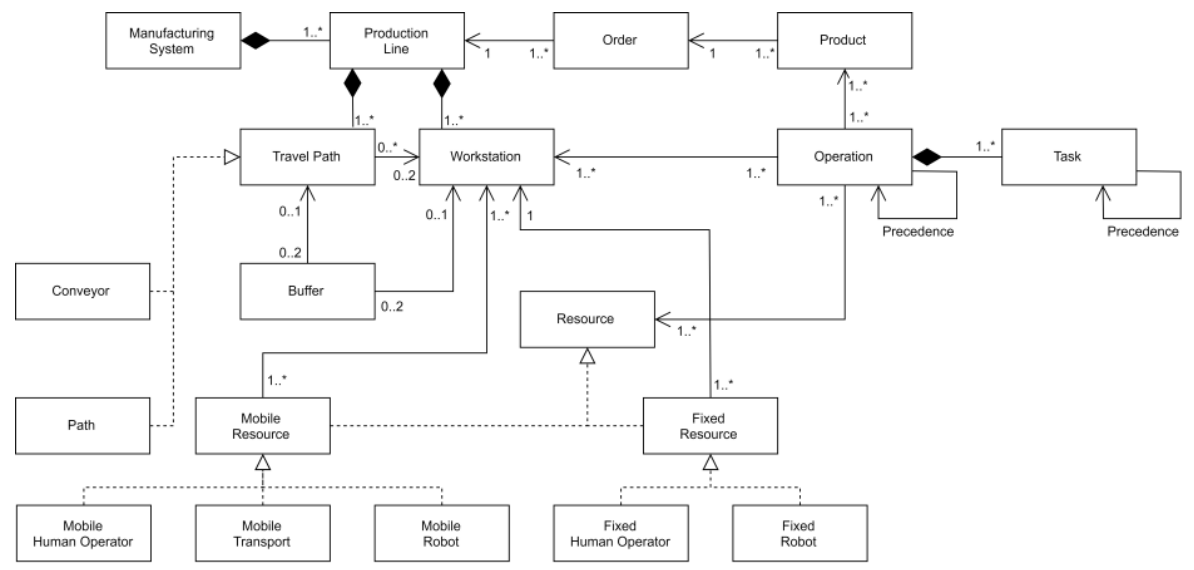

Fig. 4. U se case - D ata M odel

In a production line, production plans with a set of orders are executed. For each order, a set of products needs to be produced, and each one requires a set of operations with a given sequence and precedencies. The Data Model in Figure 4, also used by [17], represents the classes of the system, which contain specific attributes and methods.

In addition, the following data structures are required to allow running the optimization, simulation, monitoring and predictive analytics tools: (i) probability distributions of the production times of the tasks and time that a resource takes to move or transport a product from one point to another; (ii) equipment data; (iii) maintenance and production schedules; (iv) work-in-progress in the system; and (v) resources state.

\subsection{Outcomes and Potential Results}

The DSS proposed in this paper will serve as an important tool for enhancing and simplifying the production and maintenance managers' decisions relative to the WAL.

The managers can follow the current status of the factory in a single dashboard, where the real-time information is presented. This enables a faster response to malfunctions in production resources and the increase of predictive maintenance, rather than corrective maintenance, resulting in lower production stoppages and lower maintenances costs. Besides, production managers can obtain optimized decisions in terms of layout configuration, namely, how many operators to allocate to each workstation, in order to maximize the WAL productivity. Testing disruptive scenarios, such as introducing new products or production plans, and adding or removing resources, is also possible through the usage of simulation.

The outputs of this testing scenario are Key Performance Indicators (KPIs) of the line (e.g. line efficiency, resource utilization, cycle time, makespan and number of products produced). The proposed DSS can make a significant impact on the production system performance. Namely, the use case indicates that the usage of the proposed solution to test dynamic resource allocation can provide an improvement of $3 \%$ in productivity. 


\section{Conclusion}

The Industry 4.0 allows factories to generate large amounts of data coming from processes, products and components, which require the development and use of advanced tools to support the decision-making process and improve performance. These advancements are increasing the variety of approaches for DSS. It can be composed by a set of singular tools to organize and control data, and to extract knowledge in order to create inputs for the decision-makers. This paper proposes a hybrid DSS Framework that is composed by a Simulation and Optimization component, an artificial intelligence component and a visualization tool, enabled by an Open IIoT Platform.

This combination makes the proposed DSS a multi-disciplinary tool, which cannot fit into a single DSS category. The proposed DSS is considered a pillar for the digital manufacturing transformation and can be used to face a wide range of problems, considering predictive, optimization, prescriptive and simulation abilities.

The application case demonstrated the feasibility of the proposed DSS in a real case study on the aeronautics industry. The proposed solution is used to predict the probability of failure for robotic resources, to calculate the optimized scheduling considering the maintenance intervention and to estimate the expected production line performance. It can also be used to forecast the system's behaviour in other situations such as: $(i)$ introduction of new products; (ii) add, remove or change resources; and (iii) introduction of new production plans. This solution helps to speed up and support decision-making activities and to improve resource utilization and productivity of the production system.

\section{Acknowledgments}

This work has received funding from the European Union's Horizon 2020 research and innovation programme under the Grant Agreement $\mathrm{N}^{\circ} 777096$ and from SEPIN/MCTI under the 4th Coordinated Call BR-EU in CIT.

\section{References}

1. A. G. Frank, L. S. Dalenogare and N. F. Ayala, Industry 4.0 technologies: Implementation patterns in manufacturing companies, International Journal of Production Economics 210, 15 (2019)

2. F. Civerchia, S. Bocchino, C. Salvadori, E. Rossi, L. Maggiani and M. Petracca, Industrial Internet of Things monitoring solution for advanced predictive maintenance applications, Journal of Industrial Information Integration 7, 4 (2017)

3. I. Nunes and D. Jannach, A systematic review and taxonomy of explanations in decision support and recommender systems, User Modeling and User-Adapted Interaction 27 (3-5), 393 (2017)

4. M. J. Aqel, O. A. Nakshabandi and A. Adeniyi, Decision support systems classification in industry, Periodicals of Engineering and Natural Sciences 7 (2), 774 (2019)

5. H. Panetto, B. Iung, D. Ivanov, G. Weichhart and X. Wang, Challenges for the cyberphysical manufacturing enterprises of the future, Annual Reviews in Control 47, 200 (2019)

6. D. J. Power, Decision support systems: concepts and resources for managers. Greenwood Publishing Group, (2002),

7. S. W. Lin, V. F. Yu and C. C. Lu, A simulated annealing heuristic for the truck and trailer routing problem with time windows, Expert Systems with Applications 38 (12), 15244 (2011) 
8. N. Caldas, J. Sousa, S. Alcalá, E. Frazzon and S. Moniz. A simulation approach for spare parts supply chain management. In: Proceedings of the International Conference on Industrial Engineering and Operations Management, July 23-26 Pilsen, Czech Republic (To be published).

9. S. Boyd and L. Vandenberghe, Convex optimization. Cambridge university press, (2004),

10. T. Loukil, J. Teghem and D. Tuyttens, Solving multi-objective production scheduling problems using metaheuristics, European Journal of Operational Research 161 (1), 42 (2005)

11. J. Basto, J. Sousa, S. Alcalá, E. Frazzon and J. Soeiro. Optimal design of additive manufacturing supply chains. In: Proceedings of the International Conference on Industrial Engineering and Operations Management, July 23-26 Pilsen, Czech Republic (To be published).

12. M. J. Shaw and A. B. Whinston, An artificial intelligence approach to the scheduling of flexible manufacturing systems, IIE Transactions (Institute of Industrial Engineers) 21 (2), 170 (1989)

13. R. Elshawi, S. Sakr, D. Talia and P. Trunfio, Big Data Systems Meet Machine Learning Challenges: Towards Big Data Science as a Service, Big Data Research 14, 1 (2018)

14. M. I. Jordan and T. M. Mitchell, Machine learning: Trends, perspectives, and prospects, Science 349 (6245), 255 (2015)

15. T. P. Carvalho, F. A. A. M. N. Soares, R. Vita, R. d. P. Francisco, J. P. Basto and S. G. S. Alcalá, A systematic literature review of machine learning methods applied to predictive maintenance, Computers \& Industrial Engineering 137, 106024 (2019)

16. W. W. Eckerson, Performance dashboards: measuring, monitoring, and managing your business. John Wiley \& Sons, (2010),

17. R. Santos, J. Basto, S. G. S. Alcalá, E. Frazzon and A. Azevedo. Industrial IoT integrated with simulation - A digital twin approach to support real-time decision making. In: Proceedings of the International Conference on Industrial Engineering and Operations Management, July 23-26 Pilsen, Czech Republic (To be published).

18. M. Leusin, E. Frazzon, M. Uriona Maldonado, M. Kück and M. Freitag, Solving the Job-Shop Scheduling Problem in the Industry 4.0 Era, Technologies 6 (4), 107 (2018)

19. T. Wagner, C. Herrmann and S. Thiede, Industry 4.0 Impacts on Lean Production Systems, Procedia CIRP 63, 125 (2017)

20. H. Lasi, P. Fettke, H.-G. Kemper, T. Feld and M. Hoffmann, Industry 4.0, Business \& Information Systems Engineering 6 (4), 239 (2014)

21. F. Almada-Lobo, The Industry 4.0 revolution and the future of manufacturing execution systems (MES), Journal of innovation management 3 (4), 16 (2016)

22. R. Geissbauer, J. Vedso and S. Schrauf, Industry 4.0: Building the digital enterprise, Retrieved from PwC Website: https://www.pwc.com/gx/en/industries/industries4.0/landing-page/industry-4.0-building-your-digital-enterprise-april-2016.pdf, (2016)

23. P. Adolphs, H. Bedenbender, D. Dirzus, M. Ehlich, U. Epple, M. Hankel, R. Heidel, M. Hoffmeister, H. Huhle and B. Kärcher, Reference architecture model industrie 4.0 (rami4. 0), ZVEI and VDI, Status report, (2015)

24. R. Reis, F. Diniz, L. Mizioka, P. Olivio, G. Lemos, M. Quintiães, R. Menezes, F. Amadio and N. Caldas, FASTEN: an IoT platform for manufacturing. Embraer use case, MATEC Web Conf. 233, 00009 (2018) 